\title{
Expérience de thermoclastie sur quelques calcaires jurassiens
}

Cette expérience a été entreprise dans le cadre d'une étude plus générale sur la fragmentation des roches calcaires du Jura Suisse sous l'action des variations de température et des cycles gel-dégel.

D'après les données recueillies sur le terrain (A. PANCZA 1974 et 1976), les différences de températures subies par les parois et les crêtes rocheuses bien exposées du Jura sont loin d'être négligeables, même si ces différences n'atteignent pas celles relevées dans les zones arides chaudes. Les 24 et 25 janvier 1973, la température de la surface d'une paroi rocheuse située à la Vue des Alpes, non loin de Neuchâtel, s'est élevée de $1^{\circ} \mathrm{C}$. à $22^{\circ} \mathrm{C}$ en 6 heures, soit au rythme d'environ $3^{\circ} \mathrm{C} / \mathrm{h}$; la différence maxima de température entre la surface et $12 \mathrm{~cm}$ de profondeur était de $10^{\circ} \mathrm{C}$. Dans le même site, à $1280 \mathrm{~m}$ d'altitude, le 4 juillet 1973 , la température de surface est montée de 17 à $39^{\circ} \mathrm{C}$ entre 7 heures et 14 heures, soit à la vitesse de $3{ }^{\circ} \mathrm{C} / \mathrm{h}$; la différence de température entre la surface et $6 \mathrm{~cm}$ de profondeur était de $10^{\circ} \mathrm{C}$ entre 12 heures et 14 heures, et elle était de $13{ }^{\circ} \mathrm{C}$ entre la surface et $12 \mathrm{~cm}$ de profondeur aux mêmes heures.

Des valeurs importantes sont parfois citées à propos de la dégradation des monuments situés en zone tempérée à légère tendance continentale. Selon J. P. PAULY (1973) et H.F.JOWAY (1973) qui donnent des chiffres concernant le dôme des Invalides et le dôme du Val de Grâce à Paris et la tour de la cathédrale de Strasbourg, les écarts entre les températures diurnes et nocturnes peuvent atteindre 21 à $26^{\circ} \mathrm{C}$ sur les faces sud et est; la vitesse d'échauffement la plus importante est de l'ordre de $3{ }^{\circ} \mathrm{C} / \mathrm{h}$ sur les faces exposées à l'est.

Faute de pouvoir multiplier les installations expérimentales, les roches ont été placées sur un dispositif mis au point pour tester des échantillons provenant du Sahara. Il ne faut donc pas tirer des conclusions sur ce que serait l'évolution exacte des roches dans leur cadre naturel. Cependant nous avons obtenu une intéressante échelle de comportement d'une série variée de calcaires vis-à-vis de la thermoclastie et de l'humectation-dessication combinées.

Les échantillons proviennent de l'arc interne jurassien. Les roches calcaires et marno-calcaires étudiées appartiennent au Jurassique moyen, au Jurassique supérieur et au Crétacé. Afin de ne pas ébranler des échantillons, ceux-ci ont été dégagés selon les zones de faiblesse de la roche (diaclases, joints de stratification) à l'aide d'une barre à mine. Toutes les pierres retenues ont été prélevées à une certaine profondeur (au moins $40 \mathrm{~cm}$ ) par rapport à la surface des parois. Le volume des blocs utilisés varie de 1 à $1,7 \mathrm{dm}^{3}$ et le poids de 2,5 à $4 \mathrm{~kg}$ environ.

Le cycle expérimental se décompose de la manière suivante:

- chauffage pendant $43 \mathrm{mn}$ portant la surface du bloc à $65-68^{\circ} \mathrm{C}$ et la différence de température entre la surface et le cœur à environ $20^{\circ} \mathrm{C}$.

- refroidissement brutal de la surface grâce à une chute de pluie d'une durée de $2 \mathrm{mn}$ s'accompagnant d'une annulation de la différence de température entre la surface et le cœur et parfois d'une légère inversion du gradient (cœur temporairement plus chaud que la surface). Ce système entraîne l'apparition du phénomène d'humectation-dessication dont les effets sur certaines roches sont à considérer.

- réchauffement modéré pendant 10 minutes;

- refroidissement à la température ambiante.

Les échantillons ont été l'objet de 8 contrôles portant sur l'état des surfaces, sur l'état des joints de la roche, sur la création de fissures et la perte de débris.

\section{Comportement des divers faciès}

Les caractéristiques des divers faciès et les données concernant leur comportement au cours de l'essai de thermoclastie sont réunies ci-dessous:

\section{Dalle nacrée du Callovien}

Le prélèvement a été effectué dans les affleurements de la Clusette (546,23/200,65 F. 1163 Travers). La roche est un calcaire en plaquettes à cassure spathique grise, jaune-roux en surface; elle renferme des fragments d'entroques et d'échinodermes et sa porosité est de 1,6 à 1,8 .

Deux échantillons no 77 et 78 ont subi l'essai (14990 cycles). Deux fissures de l'échantillon 77 se sont accentuées pendant les 2000 premiers cycles. Mais en-

J.P. Coutard, Centre de Géomorphologie du C.N.R.S, Caen. A. Pancza, Prof., 2056 Dombresson. 


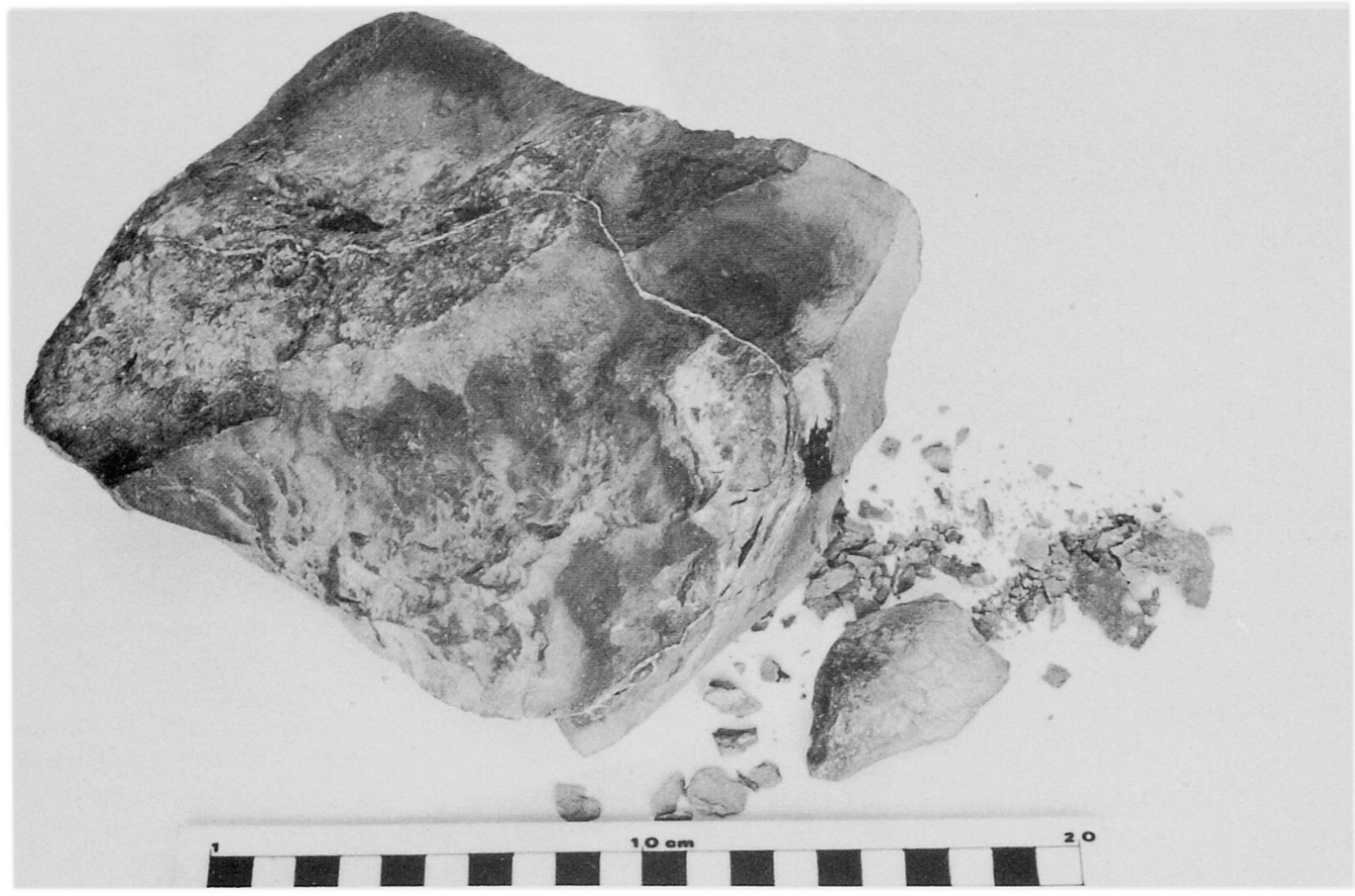

$\Delta$

1. Echantillon 714 (14 290 cycles) Marno-calcaire de l'Oxfordien

Perte d'un gros éclat selon des fissures préexistantes Zone d'écaillage sur une face latérale.
2. Echantillon 713 (14990 cycles)

Marno-calcaire grumeleux du Séquanien Zones d'écaillage sur les faces latérales.

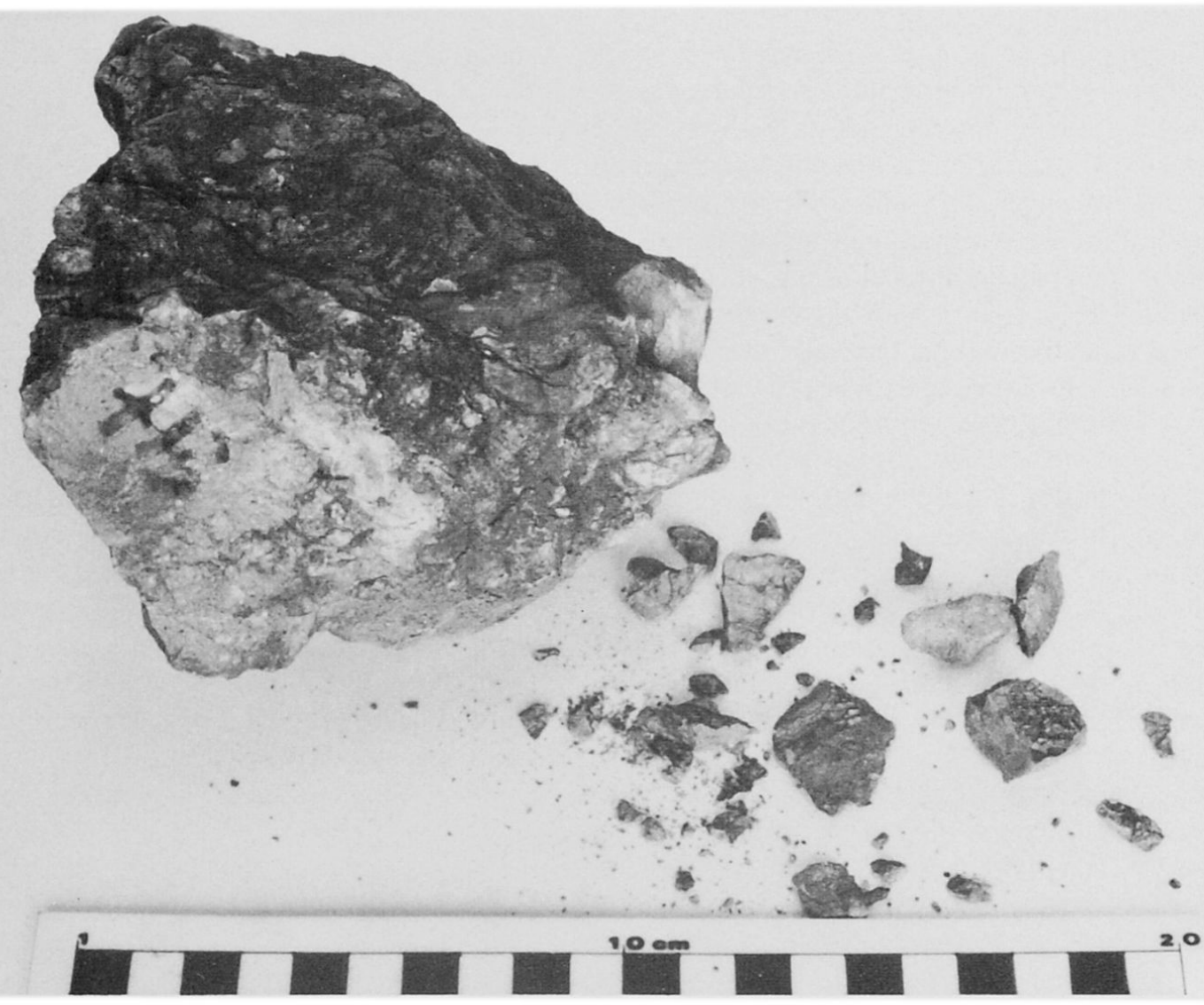




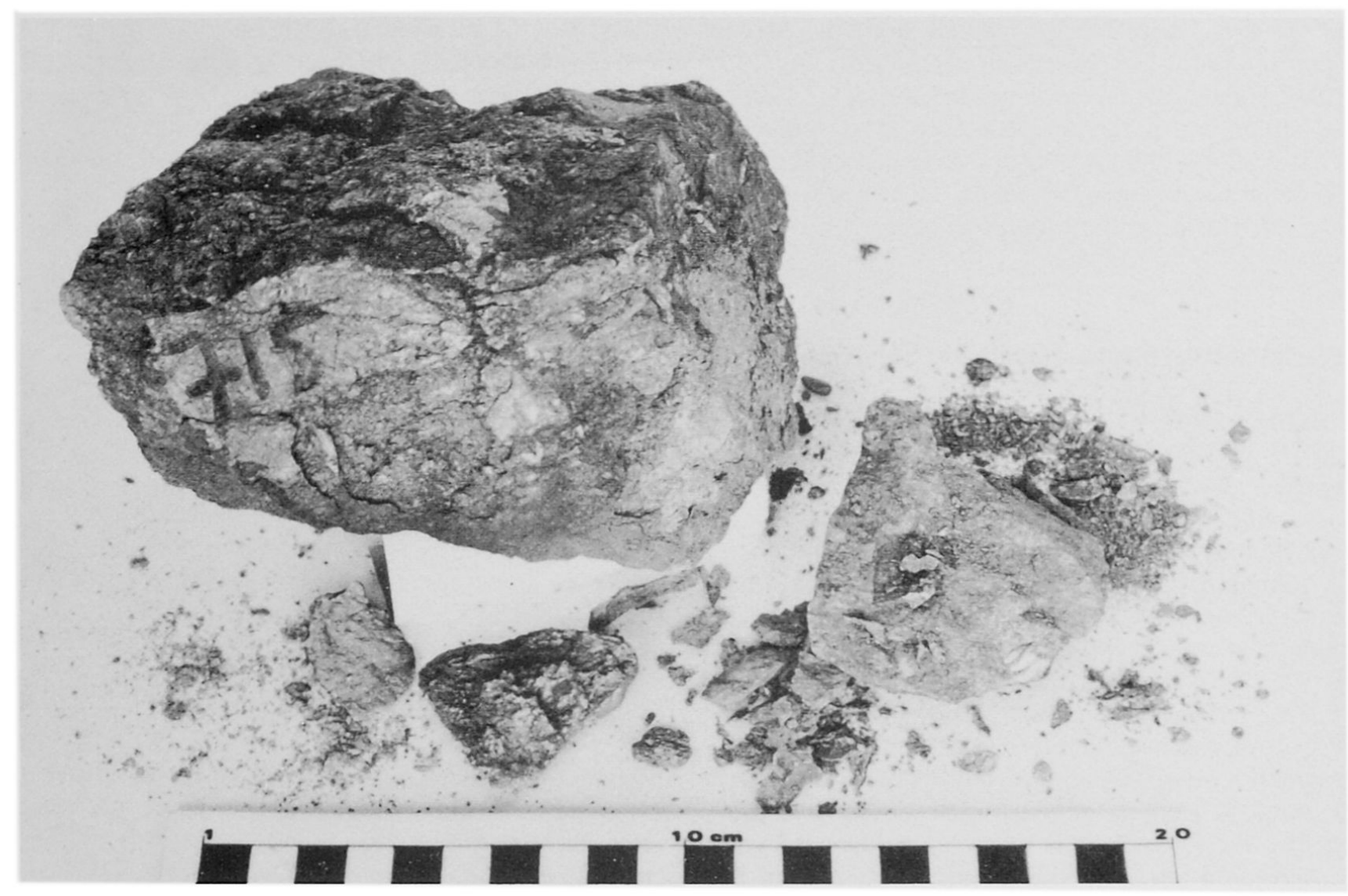

3. Echantillon 715 (14 990 cycles)

Marno-calcaire grumeleux du Séquanien

Perte de gros éclats selon des joints de la roche

Ecaillage des faces latérales amenant une véritable alvéolisation en dessous du chiffre.

suite, aucun phénomène n'est apparu sur les deux blocs calcaires. Les poids sont restés les mêmes qu'au départ soit 2960 et $2660 \mathrm{gr}$.

\section{Marno-calcaire de l'Oxfordien}

Les assises du Crêt-Pourri près de Saint-Claude $(873,84$ / 161,62 feuille 1:20000 Saint-Claude) sont constituées d'une alternance de couches de marnes et de marnocalcaires; les marnes dominent; les couches de calcaire, jaunâtre ou grisâtre, de porosité 1,6 à 2,9 sont fortement diaclasées.

Les deux échantillons no 712 et 714 ont subi 14290 cycles. Leur comportement a été différent. Sur l'échantillon 714, un écaillage a débuté entre 5000 et 8000 cycles sur les faces latérales et s'est poursuivi ensuite, la perte de poids étant de $37 \mathrm{gr}$ à la fin de l'expérience (poids initial de l'échantillon $3497 \mathrm{gr}$ ). Une fissure de l'échantillon 712 s'est marquée entre le début de l'essai et 5000 cycles, une période de stabilité est venue ensuite, puis un écaillage a commencé vers 10000 cycles sur une des faces latérales et s'est poursuivi de façon permanente. En fin d'expérience, la perte de poids était de $6 \mathrm{gr}$ (poids initial de l'échantillon: $3652 \mathrm{gr}$ ).

\section{Calcaire crayeux du Rauracien}

Le prélèvement a été réalisé au lieu dit Château Cugny à environ $5 \mathrm{~km}$ au N.W. de Saignelégier $(564,85 /$ 236,90 F. 1104 Saignelégier). Ce lieu désigne un piton rocheux qui atteint $828 \mathrm{~m}$ d'altitude. La base de l'édifice est constituée d'un calcaire massif Oxfordien surmonté de couches appartenant au Rauracien. La roche subcrayeuse, riche en faune (Térébratules) a une cassure mate blanchâtre; elle est poreuse (porosité 7 à 11 ) et sensible au gel comme le montrent les éboulis à éléments de petite taille au pied des parois. Trois échantillons 709, 710, 711 bis ont subi 14290 cycles pendant l'essai de thermoclastie. Aucun phénomène n'a été observé sur ces trois échantillons qui ont conservé leurs poids d'origine.

\section{Calcaire oolitique du Rauracien}

Les échantillons ont été pris en bordure de la route Saint-Claude-Valfin $(870,94 / 163,54$ feuille SaintClaude) à un endroit où la série se compose d'une suite de roches compactes entrecoupées par des zones moins résistantes qui donnent fréquemment de petits 
abris. La roche prélevée est blanche, oolitique, poreuse (porosité 7 à 12$)$ de résistance modérée $\left(1,5\right.$ à $\left.1,9 \mathrm{~kg} / \mathrm{cm}^{2}\right)$. Entre le début de l'expérience et 5000 cycles, l'un des échantillons a perdu un petit éclat (2 grammes), et l'échantillon 719 a vu trois petites fissures s'accentuer légèrement. Ensuite, jusqu'à la fin de l'essai (14290 cycles), plus aucune observation positive n'a pu être faite.

\section{Calcaire et marno-calcaire du Séquanien}

Les blocs ont été extraits au lieu dit La Clusette (546,18 / 200,75 F. 1163 Travers). La série marno-calcaire est entrecoupée de bancs de calcaire jaunâtre. Deux échantillons de calcaire dur, à grain grossier, jaunâtre ont été pris en bordure de la route cantonale (éch. 707 et 708) de même que trois échantillons de marno-calcaire grumeleux qui ont été prélevés à l'entrée Ouest du tunnel routier en construction (éch. 711, 713, 715). Les échantillons ont subi un nombre de cycles différent, 11450 cycles pour le no 708 et 14990 cycles pour les autres. Les échantillons de calcaire dur (707 et 708) n'ont pas subi de perte de poids et ne se sont pas fissurés. Il n'en est pas de même pour les calcaires marneux et grumeleux dont les échantillons ont vu un écaillage en fines lamelles (épaisseur 0,5 à $1 \mathrm{~mm}$, longueur 0,5 à $1 \mathrm{~cm}$ ) et une perte de fragments de forme aplatie (épaisseur 0,5 à $1,2 \mathrm{~cm}$, longueur 2 à $4 \mathrm{~cm}$ ) débuter vers le 2000 e cycle et se poursuivre sans cesse ensuite. Les échantillons dont les poids initiaux étaient de 2370,2040 , et 3035 gr ont perdu respectivement 11,42 et 231 gr. L'écaillage a lieu sur les faces latérales et inférieures des blocs alors qu'il est insignifiant sur la face supérieure.

\section{Calcaire dur à grain fin du Séquanien}

Les prélèvements ont été faits à la Côte Lambercier (543,20/200,50 F. 1163 Travers) dans une paroi rocheuse d'environ $20 \mathrm{~m}$ de haut. La roche jaune ou blanchâtre est bien stratifiée mais plus ou moins fissurée selon les strates. Les échantillons $71,72,73$ et 74 ont été pris dans une couche très résistante d'environ $20 \mathrm{~cm}$ d'épaisseur: calcaire compact à grain très fin, très peu poreux (porosité 1,1 à 2).

Au cours des 11450 cycles (éch. 71, 73, 74) ou 14990 cycles (éch. 72) aucune fissuration n'est apparue et aucune perte de poids n'a été enregistrée. Seul l'échantillon 73 a perdu un petit éclat de $5 \mathrm{gr}$ au tout début de l'expérience.

\section{Calcaire lithographique du Portlandien}

Les blocs (éch. 716, 717) proviennent d'une carrière située dans la partie occidentale de l'anticlinal de Chaumont, en bordure de la route Neuchâtel-Fenin $(559,47$ I
206,30 F. 1164 Neuchâtel). Le calcaire lithographique à taches jaunes très peu poreux (porosité 0,4 à 1,1 ) possède quelques joints d'aspect stylolithique et se présente en strates massives. Après 14990 cycles les échantillons demeuraient intacts. Toutefois quelques joints se sont accusés.

\section{Calcaire roux du Valanginien}

Les échantillons (no 75 et 76 ) ont été prélevés à $3 \mathrm{~m}$ de profondeur dans une fouille creusée au Nord de la Gare de Neuchâtel $(561,50 / 205,20)$. La roche est un calcaire zoogène légèrement ferrugineux de teinte rousse à gros grain, de porosité et de résistance moyenne (porosité 4 à $5 \%$, résistance 1,7 à $2 \mathrm{~kg} / \mathrm{cm}^{2}$ ).

Au bout de 14990 cycles les effets étaient très modérés (perte de quelques grammes de sable sur l'échantillon 75 .

\section{Conclusion}

Malgré un nombre de cycles déjà important (entre 14000 et 15000 ) pour la plupart des échantillons, les effets de la thermoclastie: fissuration, perte de grains ou de débris sont nuls ou excessivement modérés sur la majeure partie des calcaires: lithographique, oolitique, détritique dur. Seuls les marno-calcaires ont réagi au type d'essai auquel ils ont été soumis; tous ont subi très tôt, avant deux mille cycles, un écaillage qui s'est poursuivi ensuite tout au long du déroulement de l'expérience. Là, nous touchons un délicat problème d'interprétation. En effet, comme nous l'avons indiqué, l'introduction d'une chute de pluie pour assurer un refroidissement brutal de la roche a eu pour conséquence la création de cycles d'humectation-dessication: phénomène qui s'ajoute au rôle des chocs thermiques. La constatation que seuls les marno-calcaires ont subi un écaillage alors qu'ils sont les plus susceptibles de retenir l'humidité, nous incite à croire que cet écaillage tient davantage aux fluctuations d'humidité qu'aux fluctuations de température. Cela serait confirmé par une observation importante: l'écaillage est actif sur les faces latérales et inférieures qui restent longtemps humides avant de sécher et très peu développé sur la face supérieure sur laquelle l'eau ruisselle immédiatement et qui, de ce fait, s'asséche rapidement lors du réchauffement postérieur à la pluie.

Un tel écaillage en minces feuillets qui conduit à une alvéolisation large et peu profonde de certaines faces de pierres a été assez souvent observé en domaine méditerranéen sur des roches calcaires tendres ou calcaréo-marneuses. Dans notre essai, seule la perte de quelques éclats épais semble directement liée aux cycles thermiques. 


\section{Bibliographie}

COUTARD J.P., JOURnauX A. (1976): Expériences de thermoclastie, Bull. Centre Géom. C.N.R.S. Caen, no 21 , pp. 29-32.

JOURNAUX A., COUTARD J.P. (1974): Expériences de thermoclastie sur des roches siliceuses, Bull. Centre Géom. C. N. R. S. Caen, no 18, pp. 7-20.

JOWAY H.F. (1973): Etude critique d'un édifice ancien en vue de sa sauvegarde, ler coll. intern. sur la détérioration des pierres en œuvre. La Rochelle 1972. Ed. Impr. Réunies, Chambéry, pp. 57-63.
PANCZA A. (1974): Les variations de la température estivale dans une paroi rocheuse du Jura, Géogr. Helv. 1, pp. 43-46.

PANCZA A. (1979): Contribution à l'étude des formations périglaciaires dans le Jura, thèse, Univ. Neuchâtel, 187 pages.

PAULY J. P. (1973): Les procédés de simulation et leur rapport avec les phénomènes naturels, ler coll. intern. sur la détérioration des pierres en œuvre. La Rochelle 1972. Ed. Impr. Réunies, Chambéry, pp. 131-136.

\section{Literaturbesprechung}

ALKAEMPER J. et al.: Erosion Control and Afforestation in Haraz, Yemen Arab Republic. Giessener Beiträge zur Entwicklungsforschung, Schriftenreihe des Tropeninstituts der JustusLiebig-Universität Giessen, Reihe II, Band 2, 105 S., 24 Tab., 12 Fotos, 1979, DM 20.--

Ein Grossteil des nordjemenitischen Hochlandes wird seit mehreren Jahrtausenden terrassiert und intensiv kultiviert. Im letzten Jahrzehnt verzeichnete das Land eine enorm verstärkte Emigration von arbeitsfähigen Männern in die ölreichen Nachbarländer. Der Aderlass an Arbeitskräften bewirkt eine krasse Vernachlässigung der arbeitsintensiven Berglandwirtschaft. Dies führt schon nach wenigen Jahren zu irreversiblen Schäden am Kulturland; die Ackerterrassen zerfallen und fruchtbares Bodenmaterial wird weggeschwemt. Angesichts des Fehlens von Bodenschätzen muss auch in Zukunft die Landwirtschaft die Basis für jede vernünftige Entwicklung sein.

Ein interdisziplinäres Autorenkollektiv, bestehend aus einem Geomorphologen, einem Agronomen, zwei Oekologen und einem Forstökonomen, versuchte am Beispiel der Region Jabal Haraz Aufnahmen zum Erosionsprozess und zur Erosionsbekämpfung zu erbringen. Ihr Bericht dient als Grundlage zu einem im letzten Kapitel entworfenen Entwicklungsprojekt zur lokalen Waldaufforsting. Dieses Projekt zielt im wesentlichen auf die Erhaltung und Verbesserung des landwirtschaftlich genutzten Bodens und soll den
Aufbau einer planmässigen nationalen Forstwirtschaft unterstützen. Durch Förderung der privaten Brenn- und Bauholzaufforstung auf brachliegenden Ackerflächen der einzelnen Landwirtschaftsbetriebe soll langfristig der Holzraubbau unterbunden werden. Die Kosten-Ertragsanalyse zeigt, dass eine geordnete Waldbewirtschaftung eine interne Kapitalverzinsung von durchschnittlich 17 : erbringen würde. Zudem erfordert die Aufforstung einen ausserordentlich geringen Arbeitsaufwand, was dem derzeitigen Arbeitskräftemangel entgegenkommt.

Der Projektvorschlag basiert auf einer sozioökonomischen Untersuchung, wobei erstmals für die Region des Jabal Haraz detaillierte Zahlen über Nutzholzverbrauch und -gewinnung erhoben wurden. Auch von der traditionellen Vieh- und Ackerbauwirtschaft wurden eingehende Ertragsanalysen angestellt. Die Studie erbringt viel neues und reiches Datenmaterial zur ökonomischen Situation der Berglandwirtschaft, zur Abwanderung und Brachlegung. Man vermisst allerdings eine kartographische Darstellung der heutigen Landnutzung im engeren Projektbereich, in der die heute schon brachgelegten Flächen quantitativ erfasst sind. Durch die systematische Analyse ihrer Lageparameter wäre es möglich, eine "Brachlandgefährdungskarte" zu erstellen und diese als Grundlage für gezielte Massnahmen gegen den Brachlegungsprozess zu verwenden.

GH 1/81 Rudolf Schoch, Zürich 